\title{
Geld aus dem Nichts: Geldschöpfung der Banken und ihre Folgen für die Wirtschaft
}

Mathias Binswanger

\section{Der Prozess der Geldschöpfung durch Geschäftsbanken}

Wenn es darum geht, die Tätigkeit von Banken zu beschreiben, dann stellen sich die meisten Menschen Folgendes vor: Banken sammeln die Ersparnisse von Haushalten oder Unternehmen, die in einer bestimmten Periode mehr Geld verdienen als sie ausgeben wollen und vergeben diese dann wiederum als Kredite an Unternehmen oder ebenfalls Haushalte, die in einer bestimmten Periode mehr Geld ausgeben wollen, als sie verdienen, und sich deshalb Geld ausleihen. Je mehr also in einer Wirtschaft gespart wird und die Menschen Geld auf ihren Bankkonten lassen, umso mehr können die Banken dann Kredite vergeben. Die Geschäftsbanken können gemäß dieser Sichtweise nicht mehr Kredite vergeben, als das Publikum willens ist, ihnen an Ersparnissen zur Ausleihung zur Verfügung zu stellen. Banken sind somit Finanzintermediäre, deren Tätigkeit in erster Linie darin besteht, Geld von Sparern (Einlegern) zu Investoren (Kreditnehmern) zu transferieren. Dafür verlangen sie von den Kreditnehmern einen Zins, welcher den Zins, den sie selbst an die Einleger zahlen, übersteigt. Diese Differenz zwischen Soll- und Habenzinsen macht die Bankentätigkeit profitabel und sorgt dafür, dass sich Banken stets darum bemühen, weitere Ersparnisse zu akquirieren. ${ }^{1}$

Diese traditionelle Vorstellung der Bankentätigkeit ist in der Abbildung $1 \mathrm{zu}$ sammengefasst.

Diese Sichtweise erscheint auf den ersten Blick plausibel und wird in vielen Lehrbüchern zu Banken auch noch so dargestellt. Wären Banken allerdings tatsächlich Finanzintermediäre im eben beschriebenen Sinn, dann müsste man in der Realität damit rechnen, dass ihnen immer wieder die Ersparnisse ausgehen, und sie dann keine Kredite mehr vergeben können. Die Banken wären dann vergleichbar mit einer Blutbank in einem Krankenhaus. Die Bank müsste ihren Kunthias: Geld aus dem Nichts. Wie Banken Wachstum ermöglichen und Krisen verursachen, Weinheim: Wiley-VCH 2015. 
Abbildung 1: Falsche Vorstellung der Banken als Intermediäre.

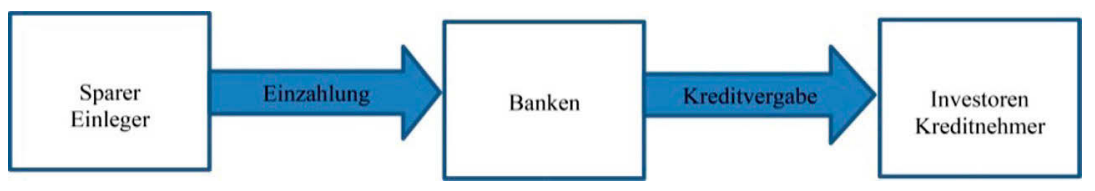

den dann genau wie eine Blutbank von Zeit zu Zeit mitteilen: >Leider haben wir im Moment keine Ersparnisse (Blut) mehr, aber Sie können sich in eine Warteliste eintragen, und wir benachrichtigen Sie dann, wenn wieder Ersparnisse bei uns eingetroffen sind.< Eine solche Mitteilung hat aber wohl noch kaum jemand von seiner Bank erhalten, aus dem einfachen Grund, weil Banken für die Kreditvergabe unmittelbar keine Ersparnisse brauchen. Sie haben stets die Möglichkeit, weitere Kredite zu vergeben, solange weitere Nachfrage nach Krediten existiert und die potentiellen Kreditnehmer als kreditwürdig eingestuft werden. Ein Finanzintermediär, der nur Ersparnisse sammelt und diese dann wieder ausleiht, wäre dazu nicht in der Lage.

Von der liebgewonnenen Vorstellung einer Bank als Finanzintermediär müssen wir uns deshalb lösen. Andernfalls können wir weder die Tätigkeit von Banken noch die Wirkung des Geldes in einer modernen Wirtschaft verstehen. Banken wirken zwar auch als Finanzintermediäre, wenn Investment-Banken Unternehmen dabei helfen, Käufer für die von den Unternehmen emittierten Wertpapiere zu finden. Sobald es aber um die Kreditvergabe selbst geht, ist eine Bank >Geldproduzent « und nicht Finanzintermediär. Auch einzelne Zentralbanken haben dies mittlerweile erkannt. Die Deutsche Bundesbank betont in der auf ihrer Homepage zu findenden Broschüre Geld und Geldpolitik ${ }^{2}$ ausdrücklich die Rolle der Banken als geldschöpfende Institutionen. Und die Bank of England erklärt in einem Beitrag in ihrer Zeitschrift Quarterly Bulletin zu Beginn des Jahres 2014 ausführlich, wie Geschäftsbanken über Kreditvergabe Geld schaffen. ${ }^{3}$ Dieser Prozess wird im folgenden Kapitel beschrieben.

Der Prozess der Geldschöpfung in der heutigen Wirtschaft wird im Wesentlichen durch den Satz >Kredite schaffen Einlagen beschrieben oder, wie es im englischen Original heißt: >Loans make depositsı. In einer modernen Wirtschaft entsteht Geld hauptsächlich durch die Kreditvergabe der Geschäftsbanken. Wann

2 Deutsche Bundesbank: Geld und Celdpolitik, Frankfurt a.M.: Deutsche Bundesbank 2019, https://www.bundesbank.de/de/publikationen/schule-und-bildung/geld-und-geldpoliti k-606038 (letzter Abruf 20.06.2020).

3 McLeay, Michael/Radia, Amar/Thomas, Ryland: »Money Creation in the Modern Economy«, in: Bank of England Quarterly Bulletin Q1 (2014), S. 14-27. 
immer eine Geschäftsbank entscheidet, dass ein Bankkunde kreditwürdig ist und sie ihm deshalb einen Kredit gibt, dann wird der entsprechende Betrag dem Konto des Bankkunden gutgeschrieben. In dem Moment, wo die Gutschrift erfolgt, erhöht sich dann entsprechend auch die Geldmenge, die heute zum größten Teil aus Einlagen auf Bankkonten besteht. Diese Einlagen bilden das Giralgeld, welches materiell gar nicht in Erscheinung tritt und nur als Zahl auf einem Konto existiert. Aus diesem Grund kann man auch von >Geldschöpfung aus dem Nichts< sprechen, da es keine physische Produktion braucht, um dieses Geld zu schaffen. Das heißt nicht, dass eine Geschäftsbank Geld ohne irgendwelche Kosten herstellen kann. Sie muss einerseits die Kreditwürdigkeit eines potenziellen Kunden abklären und auch die ganze Infrastruktur für Zahlungsvorgänge zur Verfügung stellen. Aber die Geldschöpfung selbst erfolgt ohne unmittelbaren Ressourcen- und Arbeitsaufwand. Der britische Ökonom und Staatsmann Lawrence Lee Bazley Angas schrieb deshalb schon 1937: "Das moderne Bankensystem erzeugt Geld aus dem Nichts. Dieser Prozess ist vielleicht der erstaunlichste Taschenspielertrick, der jemals erfunden wurde. $\aleph^{4}$ Und die Deutsche Bundesbank schreibt in ihrer schon erwähnten Broschüre Geld und Geldpolitik: »Der Geldschöpfungsprozess erscheint wie Zauberei.«

Allerdings handelt es sich bei der Geldschöpfung um einen letztlich leicht nachzuvollziehenden Prozess, der sich in wenigen Sätzen beschreiben lässt. Banken zahlen den Kredit an einen Kunden mit Sichteinlagen (Forderung gegenüber der Bank), die nicht vorher von Sparern bei ihnen deponiert wurden, sondern die sie selbst schaffen. Dieses Geld wird, wie wir gesehen haben, nicht physisch produziert, sondern virtuell einem Bankguthaben gutgeschrieben. Wird nun umgekehrt ein Kredit zurückgezahlt, verringert sich die Geldmenge wieder um den entsprechenden Betrag, da das Geld dann wieder vom Guthaben des Bankkunden abgebucht wird. Worauf es letztlich ankommt, ist die Nettokreditvergabe. Werden mehr neue Kredite vergeben, als Kredite zurückbezahlt werden, dann erhöht sich die Geldmenge. Und in einer wachsenden Wirtschaft ist das längerfristig immer der Fall, da ständig mehr Kredite für die Finanzierung von Investitionen gebraucht werden.

Physisch in Erscheinung tritt Geld erst dann, wenn wir es bar in Form von Banknoten vom Konto abheben bzw. am Bankomat beziehen, worauf wir als Besitzer eines Sichtguthabens ein Recht haben. Solange wir einer Bank vertrauen, Geld bei Bedarf in bar auszahlen zu können, wollen wir Bargeld kaum in großen Mengen beziehen. Wir ziehen es oft vor, die Guthaben selbst als Zahlungsmittel zu verwenden, weil dies einfacher und sicherer ist. So nimmt der bargeldlose Zahlungsverkehr immer mehr zu, während Barzahlungen über lange Zeit rückläufig 
waren. Da sie aber den Vorteil der Anonymität besitzen, haben sie in neuester Zeit wieder etwas an Attraktivität gewonnen.

Anerkennen wir die Rolle der Banken als geldschöpfende Institutionen, dann lässt sich der Prozess der Kreditvergabe wie in Abbildung 2 darstellen.

Abbildung 2: Richtige Vorstellung von Banken als geldschöpfende Institutionen.

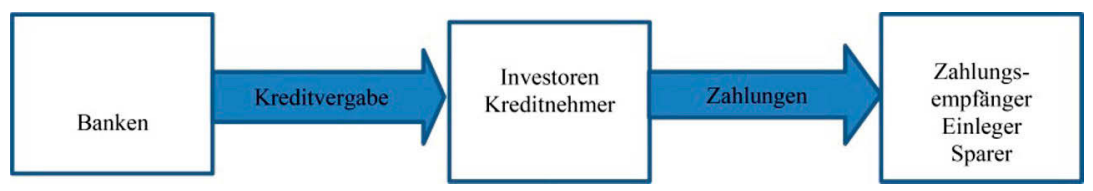

Die Kreditvergabe beginnt nicht mit dem Sparer, der sein Geld bei der Bank vorbeibringt, sondern mit der Bank selbst, die durch die Vergabe eines Kredites neues Geld schafft und auf diese Weise das Konto des Kreditnehmers füllt. Dieser verwendet das Geld dann wiederum, um damit Güter oder Dienstleistungen zu kaufen bzw. Projekte zu finanzieren, wobei das Geld von seinem Konto abgebucht und den Konten der Verkäufer der Güter und Dienstleistungen gutgeschrieben wird. Auch die neuen Geldbesitzer werden das Geld zumindest teilweise wieder ausgeben, so dass die Konten immer neuer Zahlungsempfänger gefüllt werden, während es von den Konten der Zahler jeweils wieder verschwindet. Am Ende des Jahres ist das Geld dann auf dem Guthaben irgendeines Zahlungsempfängers, der es nicht im gleichen Jahr wieder ausgeben wird. In der volkswirtschaftlichen Gesamtrechnung wird dieser Betrag als Ersparnis verbucht. Diese Ersparnis hat aber nicht die Finanzierung des Kredits ermöglicht, sondern es ist gerade umgekehrt. Die Vergabe des Kredits durch die Geschäftsbank hat neues Geld geschaffen, welches am Ende des Jahres dann als Ersparnis gezählt wird, da es sich um Einnahmen in einem Jahr handelt, die nicht im gleichen Jahr wieder ausgegeben werden. Dies entspricht der Definition von Sparen in der volkswirtschaftlichen Gesamtrechnung.

Vergibt eine Geschäftsbank einen Kredit an einen Kunden, dann ist dieser Vorgang in der Bankbilanz und in der Bilanz des Kunden folgendermaßen verbucht: 
Tabelle 1: Eine Bank vergibt einen Kredit in der Höhe von 100.000 Euro an einen Kunden.

\begin{tabular}{|l|l|}
\hline \multicolumn{2}{|l|}{ Bilanz der Geschäftsbank } \\
\hline Aktiven: & Passiven: \\
\hline Kredit +100.000 & Einlagen +100.000 \\
\hline \multicolumn{2}{|l}{} \\
\hline Bilanz des Bankkunden & Passiven: \\
\hline Aktiven: & Kredit +100.000 \\
\hline Einlagen +100.000 &
\end{tabular}

Zeitgleich mit der Entstehung des Kredits auf der Aktivseite der Bankbilanz entsteht eine neue Einlage von 100.000 Euro auf dem Konto des Kreditnehmers, die auf der Passivseite der Bankbilanz aufgeführt ist. Diese neu geschaffenen Einlagen von 100.000 Euro erhöhen die Geldmenge um denselben Betrag. Der Kreditnehmer hat jetzt 100.000 Euro mehr auf seinem Bankguthaben, was sich auf der Aktivseite seiner Bilanz niederschlägt. Allerdings schuldet er diesen Betrag gleichzeitig der Bank, da er den Kredit irgendwann zurückzahlen muss, so dass der Kredit auf der Passivseite seiner Bilanz steht.

Wird nun umgekehrt der Kredit von 100.000 Euro wieder an die Geschäftsbank zurückbezahlt, dann verschwindet sowohl der vergebene Kredit als auch die damit verbundene Einlage auf dem Konto des Kreditempfängers und die Geldmenge schrumpft um die 100.000 Euro. In der Bankbilanz und in der Bilanz des Kunden wird dies folgendermaßen verbucht:

Tabelle 2: Rückzahlung eines Kredits in der Höhe von 100.000 Euro von einem Kunden.

\begin{tabular}{|l|l|}
\hline \multicolumn{2}{|l|}{ Bilanz der Geschäftsbank } \\
\hline Aktiven: & Passiven: \\
\hline Kredit -100.000 & Einlagen -100.000 \\
\hline \multicolumn{2}{|l|}{} \\
\hline Bilanz des Bankkunden & Passiven: \\
\hline Aktiven: & Kredit-100.000 \\
\hline Einlagen -100.000
\end{tabular}

Werden also innerhalb eines Zeitraums mehr neue Kredite vergeben, als umgekehrt zurückbezahlt werden, dann erhöht sich die Geldmenge in diesem Zeitraum.

Geld entsteht allerdings nicht nur durch die Vergabe von Krediten. Geschäftsbanken schaffen auch Geld durch den Kauf von Aktiven wie Wertpapieren, Devisen, Gold oder Grundstücken bei Nichtbanken. Verkauft zum Beispiel ein Unternehmen 
Obligationen an eine Bank, dann bezahlt die Bank diese Wertpapiere wiederum mit Geld, das sie im Moment der Bezahlung selbst schafft. Das heißt, sie schreibt dem Konto des Unternehmens einen Betrag gut, genauso wie sie dies tun würde, wenn sie dem Unternehmen einen Kredit vergäbe. Auf diese Weise steigt die Geldmenge um den Betrag an, der für den Kauf der Obligationen bezahlt wurde. Umgekehrt wird Geld vernichtet, wenn die Bank dem Unternehmen Obligationen verkauft. In diesem Fall bezahlt das Unternehmen für die Obligationen mit seinem Konto bei der Bank, wo der Betrag entsprechend abgebucht wird und die Geldmenge sinkt. Solange also Banken mehr Aktiven von Nichtbanken kaufen, als sie umgekehrt Aktiven an diese verkaufen, wird netto Geld geschöpft und die Geldmenge steigt. Aus diesem Grund stehen die Kredite in den Bankbilanzen nicht immer in einem exakten Verhältnis zu den Einlagen und damit zur Geldschöpfung, da diese auch durch Kauf und Verkauf von Aktiven beeinflusst wird.

Im folgenden Beispiel nehmen wir an, dass ein Kunde seiner Bank ein Wertpapier im Wert von 100.000 Euro verkauft. Dieser Vorgang wird folgendermaßen bilanziert:

Tabelle 3: Eine Bank kauft ein Wertpapier im Wert von 100.000 Euro von einem Kunden.

\begin{tabular}{|l|l|}
\hline \multicolumn{2}{|l|}{ Bilanz der Geschäftsbank } \\
\hline Aktiven: & Passiven: \\
\hline Wertpapiere +100.000 & Sichtguthaben +100.000 \\
\hline \multicolumn{2}{|l}{} \\
\hline Bilanz des Bankkunden & Passiven: \\
\hline Aktiven: & Sichtguthaben +100.000 \\
\hline Wertpapiere -100.000 & \\
\hline
\end{tabular}

Verkauft die Geschäftsbank im Gegensatz zum eben dargestellten Fall das Wertpapier an einen Kunden, dann kehren sich in dem eben dargestellten Fall alle Vorzeichen um.

\section{Die Rolle der Zentralbank im Geldschöpfungsprozess}

Aus den bisherigen Ausführungen geht klar hervor, dass Geschäftsbanken unmittelbar keine Ersparnisse brauchen, um Kredite zu vergeben oder Aktiven käuflich zu erwerben. Aber warum sind dann die einzelnen Banken doch daran interessiert, dass wir Konten gerade bei ihnen eröffnen und nicht bei einer anderen Bank? Warum bezahlen sie uns im Normalfall sogar noch einen Zins dafür, obwohl sie diese bei ihnen deponierten Gelder scheinbar gar nicht brauchen? 
Hier müssen wir unterscheiden zwischen einer einzelnen Geschäftsbank und dem Bankensystem als Ganzes. Für das gesamte Bankensystem spielt es keine Rolle, bei welcher Bank die Kunden ein Konto haben. Aus der Perspektive einer einzelnen Bank sieht dies aber ganz anders aus. Werden beispielsweise verstärkt Konten bei einer Bank A aufgelöst und wird das Geld dann stattdessen auf Konten einer anderen Bank B einbezahlt, dann wird dies für die Bank A schnell zu einem Problem, da sie die entsprechenden Beträge an die Bank B überweisen muss. Diese Schuld kann aber nicht mit Geldschöpfung beglichen werden, indem die Bank A der Bank B einfach den entsprechenden Betrag auf einem Konto gutschreibt. Die Zahlungen der Banken untereinander müssen mit einem Zahlungsmittel erfolgen, welches die Geschäftsbanken nicht selbst schaffen können. Dieses Zahlungsmittel sind die Reserven, womit die Beträge bezeichnet werden, welche sich auf den Guthaben der Geschäftsbanken bei der Zentralbank befinden.

Die Konten der Geschäftsbanken bei der Zentralbank dienen als ClearingSystem zwischen den Geschäftsbanken. Täglich nehmen viele Bankkunden Zahlungen vor, bei denen sie Geld von ihren Konten bei einer bestimmten Geschäftsbank auf die Konten anderer Geschäftsbanken überweisen. So werden etwa Gelder von Konten bei der Bank A auf Konten bei der Bank B überwiesen und genauso auch von Konten bei der Bank B auf Konten bei der Bank A. Am Ende des Tages wird dann der Saldo aus den Überweisungen zwischen den beiden Banken ermittelt. Wurde mehr Geld von Bank A an die Bank B überwiesen als umgekehrt, dann muss die Bank A die Differenz dadurch begleichen, dass sie entsprechend Reserven von ihrem Konto bei der jeweiligen Nationalbank an die Bank B überweist. Und je mehr bei einer Bank Gelder an andere Banken abfließen, umso mehr Reserven braucht sie, um die durch den Abfluss entstandenen Verpflichtungen an andere Banken begleichen zu können. Umgekehrt erhält eine Bank, der Giralgeld zufließt, auf diese Weise zusätzliche Reserven, die sie dann zum Bezug von Bargeld oder zur Erfüllung der Mindestreservepflicht verwenden kann.

Damit wird klar, warum Geschäftsbanken im Normalfall interessiert sind, dass Kunden Geld auf Guthaben bei ihnen und nicht auf Guthaben bei einer anderen Bank halten, obwohl dieses Geld für die unmittelbare Kreditvergabe nicht gebraucht wird. Ein Verlust von Kundengeldern führt zu einem zusätzlichen Bedarf an Reserven, die sich eine Geschäftsbank normalerweise nicht gratis beschaffen kann. Sie muss dafür einen Zins zahlen, egal ob sie sich die zusätzlichen Reserven durch den Verkauf von Wertpapieren an die Zentralbank beschafft oder von anderen Geschäftsbanken ausleiht (siehe weiter unten). Auf diese Weise wirkt sich ein Abfluss von Kundengeldern negativ auf die Profitabilität des Bankengeschäfts aus.

Wie wirkt es sich nun in der Bilanz einer Geschäftsbank A aus, wenn Geld von einem Konto bei dieser Bank auf das Konto einer andern Geschäftsbank B überwiesen wird? Dies zeigt die folgende Darstellung: 
Tabelle 4: Überweisung von 100.000 Euro von einer Geschäftsbank A an eine Geschäftsbank $B$.

\begin{tabular}{|l|l|}
\hline \multicolumn{2}{|l|}{ Bilanz der Geschäftsbank A } \\
\hline Aktiven: & Passiven: \\
\hline Reserven -100.000 & Einlagen -100.000 \\
\hline \multicolumn{2}{|l}{} \\
\hline Bilanz der Geschäftsbank B & Passiven: \\
\hline Aktiven: & Einlagen +100.000 \\
\hline Reserven +100.000 &
\end{tabular}

Wir sehen, dass die Überweisung von Geld von einem Konto von der Bank A zur Bank B zur Folge hat, dass die Bank A gleichzeitig denselben Betrag von ihrem Konto bei der Zentralbank an die Bank B überweisen muss. Das Clearing-System sorgt dafür, dass nicht jede Überweisung von Kundengeldern zwischen Bank A und B auch zu einer entsprechenden Überweisung von Reserven führt. Nur die am Ende des Tages verbleibende Differenz zwischen allen Überweisungen von Bank A $\mathrm{zu}$ Bank B und von Bank B zu Bank A wird dann über die Reserven beglichen.

Wir können also festhalten: Eine Geschäftsbank kann über Kreditvergabe oder den Kauf von Aktiven (insbesondere Wertpapiere) Giralgeld schaffen, ohne dass vorher Ersparnisse auf dem Konto der Bank einbezahlt wurden. Die Einlagen werden zusammen mit dem Kredit oder dem Kauf geschaffen und würden sonst gar nicht existieren. Trotzdem ist aber eine einzelne Bank daran interessiert, dass möglichst viele Kunden Geld gerade auf ihren Konten und nicht auf den Konten von anderen Banken halten. Ein Zufluss von Giralgeld von anderen Banken bedeutet nämlich gleichzeitig einen Zufluss von Reserven, für welche andernfalls Zins bezahlt werden müsste. Und umgekehrt bedeutet ein Abfluss von Kundengeldern für eine einzelne Bank einen Verlust von Reserven. Diese muss sie sich dann entweder von der Zentralbank oder von anderen Geschäftsbanken wieder beschaffen, wofür aber ein Zins fällig wird.

Wozu, wird man sich jetzt vielleicht fragen, braucht es überhaupt noch Zentralbanken, wenn doch Geschäftsbanken selbst Geld schaffen können? Und wie können Zentralbanken die Geldschöpfungstätigkeit der Banken beeinflussen?

Der Einfluss der Zentralbanken auf den Geldschöpfungsprozess ergibt sich dadurch, dass Banken Reserven, das heißt Geld auf ihren Konten bei der Zentralbank brauchen. Für diesen Bedarf an Reserven gibt es drei Gründe. Erstens verwenden Bankkunden nach wie vor Bargeld für Zahlungszwecke. Mit der Menge an Giralgeld, welches durch die Geschäftsbanken geschaffen wird, steigt deshalb auch der Bedarf an Bargeld, da im Durchschnitt ein bestimmter Prozentsatz des Giralgeldes in Form von Banknoten vom Konto abgehoben wird. Für den Bezug dieses 
Bargeldes brauchen die Geschäftsbanken die Zentralbank, denn diese besitzt ein vom Staat vergebenes Monopol für dessen Herstellung. Wenn eine Geschäftsbank beispielsweise zusätzliches Bargeld im Umfang von 1 Million von der Zentralbank beziehen möchte, dann geht dies nur, wenn sie vorher auch Reserven in der Höhe von 1 Million besitzt. Denn die Bezahlung für das Bargeld erfolgt dadurch, dass die 1 Million Euro vom Konto der Geschäftsbank abgebucht werden, sobald ihr die entsprechende Menge an Bargeld geliefert wird. Der Bedarf an Bargeld führt so zu einem zusätzlichen Bedarf an Reserven.

Zweitens müssen Geschäftsbanken in vielen Ländern sogenannte Mindestreserven bei der Zentralbank halten. Schaffen Banken zusätzliches Geld, dann sind sie stets verpflichtet, den sogenannten Mindestreservesatz einzuhalten. Die Summe der Guthaben der Geschäftsbanken bei der Zentralbank darf im Verhältnis zu der Summe der Guthaben der Bankkunden bei der Geschäftsbank nicht unter einen bestimmten Prozentsatz fallen. Beträgt der Mindestreservesatz für Sichtguthaben beispielsweise 2 Prozent und hat eine Geschäftsbank insgesamt Einlagen von 100 Millionen Franken auf ihren Sichtguthaben, dann muss sie mindestens 2 Millionen Reserven auf ihrem Konto bei der Zentralbank haben. Je mehr Geld die Geschäftsbanken schaffen, umso mehr Reserven müssen sie sich deshalb von der Zentralbank beschaffen.

Drittens werden Reserven auch für Zahlungen der Geschäftsbanken untereinander benötigt, welche über Konten der Geschäftsbanken bei der Zentralbank abgewickelt werden. Also braucht eine Geschäftsbank täglich ausreichend Reserven, um allfällige Zahlungen an andere Geschäftsbanken vornehmen zu können. Je mehr Giralgeld geschaffen wird, umso größere Beträge werden Bankkunden von Konten ihrer Bank auf Konten anderer Banken überweisen. Dies führt bei den Banken, die am Ende des Tages einen Nettoabfluss von Geldern zu verzeichnen haben, zu einer zusätzlichen Nachfrage an Reserven.

Aus den drei genannten Gründen führt Geldschöpfung bei den Geschäftsbanken zu einem entsprechenden Mehrbedarf an Reserven bei der Zentralbank. Zwar können Geschäftsbanken sich auch untereinander auf dem Interbankenmarkt Reserven ausleihen, und eine einzelne Geschäftsbank hat immer die Möglichkeit, sich Reserven entweder bei einer anderen Geschäftsbank oder bei der Zentralbank auszuleihen. Verzeichnet etwa die Bank A am Ende des Tages einen Nettoabfluss an Reserven, weil netto Geld von ihren Konten auf Konten anderer Banken abgebucht wurde, dann entstehen bei anderen Banken Überschussreserven, die sie dann der Bank A auf dem Interbankenmarkt gegen Zins wieder ausleihen können. Generell wird eine Geschäftsbank mit dem Bedarf an zusätzlichen Reserven diese solange bei anderen Geschäftsbanken ausleihen, wie sie dort zu einem geringeren Zinssatz als bei der Zentralbank bezogen werden können. Das Geschäftsbankensystem als Ganzes kann hingegen seinen Bestand an Reserven nur dann ausweiten, wenn die Zentralbank zusätzliche Reserven zur Verfügung stellt. 
Die Reserven und das Bargeld, welche die Geschäftsbanken bei der Zentralbank beziehen können, stellen somit Formen von Geld dar, welche durch die Zentralbank und nicht durch die Geschäftsbanken geschaffen werden. Dieses Zentralbankengeld (auch als Notenbankgeldmenge oder monetäre Basis bezeichnet) setzt sich aus den Reserven (Konten der Geschäftsbanken bei der Zentralbank plus Bargeldbestand der Geschäftsbanken) und dem in der Wirtschaft umlaufenden Bargeld zusammen und wird häufiger auch als Geldmengenaggregat Mo bezeichnet. Diese Geldmenge Mo ist normalerweise nur ein Bruchteil der Geldmengen M1, M2 und M3, welche die tatsächlich in der Wirtschaft für Zahlungen und Wertaufbewahrung existierende Geldmenge erfassen. Das Zentralbankengeld steht auf der Passivseite der Zentralbankenbilanz, da es eine Verbindlichkeit gegenüber den Geschäftsbanken (Reserven der Banken) oder gegenüber den Nichtbanken (Bargeld in den Händen der Nichtbanken) ist. Allerdings handelt es sich beim Bargeld (von der Zentralbank herausgegebene Banknoten) um eine nicht rückzahlbare und somit sewige Verbindlichkeit bzw. Schuld. Heutige Zentralbanken sind nicht mehr verpflichtet, dieses Bargeld in Gold umzutauschen, so wie dies während der Zeiten des Goldstandards der Fall war.

Die Geldschöpfung der Zentralbank selbst ist somit im Unterschied zur Geldschöpfung der Geschäftsbanken nicht durch eine Pflicht zum Umtausch in ein anderes Zahlungsmittel beschränkt. Während Geschäftsbanken verpflichtet sind, das von ihnen geschaffene Giralgeld auf Wunsch der Kunden in Bargeld umzutauschen, muss die Zentralbank das von ihr geschaffene Zentralbankengeld in nichts anderes mehr umtauschen. Allerdings hat die Zentralbank im Unterschied zu Geschäftsbanken auch keinen Anreiz, möglichst viel Geld zu schaffen, da ihr Hauptziel nicht darin besteht, Gewinne zu machen. Die Geldschöpfung der Zentralbank ist durch den in der Verfassung verankerten Auftrag beschränkt, die Inflationsrate unter zwei Prozent zu halten.

Der entscheidende Einfluss der Zentralbank auf die Geldschöpfungstätigkeit der Banken ergibt sich dadurch, dass die Zentralbank die Bedingungen festlegen kann, zu welchen Geschäftsbanken zusätzliche Reserven beziehen können. Diese erhalten sie nämlich unter normalen Bedingungen nicht gratis, sondern sie müssen der Zentralbank dafür einen Zins bezahlen Dieser Zins wird dann ebenfalls von den Konten der Geschäftsbanken bei der Zentralbank abgebucht. Die Zinszahlung muss also mit Zentralbankengeld erfolgen, welches die Banken nicht selbst schaffen können. Ist dieser Zins hoch, dann ist die Beschaffung zusätzlicher Reserven teuer und die Geschäftsbanken geben die hohen Zinsen an ihre Kunden weiter, indem sie für ihre Kredite ebenfalls höhere Zinsen verlangen. Auf diese Weise steigen die Zinsen in der gesamten Wirtschaft, was zu einem Rückgang der Nachfrage nach Krediten und damit zu einer Bremsung der Geldschöpfung führt. Umgekehrt kann die Zentralbank durch eine Zinssatzsenkung Reserven für die Banken verbilligen, was tendenziell auch tiefere Zinsen für Bankkredite bewirkt. Dies kann dann 
eine Mehrnachfrage nach Krediten auslösen, wodurch sich die Geldschöpfung beschleunigt.

\section{Die Auswirkungen der Geldschöpfung auf die Wirtschaft}

Bisher wurde dargestellt, wie Geld durch Geschäftsbanken geschaffen wird und wie Zentralbanken diesen Prozess zu kontrollieren versuchen. Im Folgenden geht es um die fundamentale Frage, welche Auswirkungen diese Geldschöpfung auf die Wirtschaft hat. Dies ist eine der umstrittensten Fragestellungen der ökonomischen Theorie, welche Ökonomen seit Hunderten von Jahren beschäftigt und häufig in die Irre geführt hat. Denn solange man den Geldschöpfungsprozess der Geschäftsbanken nicht richtig erfasst bzw. ignoriert, lassen sich die Auswirkungen einer Erhöhung der Geldmenge in der Wirtschaft nicht nachvollziehen. Wie wir sehen werden, ist die Möglichkeit der Geldschöpfung eine Voraussetzung für andauerndes Wirtschaftswachstum, welches sonst gar nicht finanzierbar wäre.

Da Geld über die Kreditvergabe bzw. durch den Kauf von Aktiven der Geschäftsbanken geschaffen wird, hängt die Auswirkung der Geldschöpfung in der Wirtschaft ganz entscheidend davon ab, was die Kunden der Bank mit dem neu geschaffenen Geld machen. Dabei lassen sich die folgenden drei grundsätzlichen Möglichkeiten unterscheiden:

1. Reales Wachstum: Geld wird produktiv verwendet zur Finanzierung eines Mehreinsatzes bzw. Verbesserung der Produktionsfaktoren Arbeit und Realkapital, was eine Ausdehnung bzw. Veränderung der Produktion bewirkt.

Unternehmen, die sich von einer Geschäftsbank Geld ausleihen, verwenden das Geld dafür, neue Maschinen oder Anlagen zu kaufen, die Beschäftigung $\mathrm{zu}$ erweitern und die Produktionsprozesse $\mathrm{zu}$ verbessern, so dass sie in $\mathrm{Zu}$ kunft mehr oder auch neue Arten von Gütern und Dienstleistungen produzieren können. Das zusätzlich in der Wirtschaft umlaufende Geld ermöglicht dann sowohl eine Zunahme der gesamtwirtschaftlichen Nachfrage als auch des gesamtwirtschaftlichen Angebots. In der Realität sind allerdings nicht alle finanzierten Investitionsprojekte erfolgreich und die Finanzierung nicht erfolgreicher Investitionsprojekte führt zu Inflation, da den erhöhten Ausgaben keine Mehrproduktion gegenüber steht. Solange aber eine Mehrheit der Investitionsprojekte erfolgreich ist, bewirken Investitionen in Realkapital genauso wie die Schaffung von neuen Arbeitsplätzen in erster Linie reales Wirtschaftswachstum.

Nur teilweise produktiv sind Hypothekarkredite. Dienen sie der Finanzierung des Baus von Immobilien, dann führen sie über einen gewissen Zeitraum auch zu einer Zunahme der Produktion. Dienen die Hypothekarkredite hingegen 
nur zum Erwerb von schon bestehenden Liegenschaften, dann führt dies nicht zu einer Zunahme der Produktion. Mehr Hypothekarkredite führen in diesem Fall tendenziell zu einer Zunahme der Immobilienpreise (siehe Punkt 3).

2. Inflation bei Gütern und Dienstleistungen: Geld wird verwendet zur Finanzierung des Kaufes von bereits existierenden Gütern und Dienstleistungen.

Wird neu geschaffenes Geld für den Kauf von bereits früher produzierten Gütern oder Dienstleistungen eingesetzt, dann erhöht sich die Geldmenge, ohne dass irgendetwas Zusätzliches hergestellt wird. In diesem Fall führt die Geldschöpfung zu Inflation, da sich die gesamtwirtschaftliche Nachfrage erhöht, aber das Angebot konstant bleibt. Eine solche nicht produktive Verwendung ergibt sich am offensichtlichsten bei Konsumkrediten, wo nur zusätzliche Nachfrage finanziert wird. Aber auch Kredite an Unternehmen und Geldschöpfung durch Aufkauf von Staatschulden können zu nicht produktiver Geldverwendung führen, wenn damit keine zusätzliche Produktion finanziert wird.

3. Inflation auf Finanzmärkten; Geld wird zum Kaufvon Wertpapieren (vor allem Aktien) oder Immobilien verwendet.

Neu geschaffenes Geld kann auch zum Kauf von Wertpapieren oder Immobilien verwendet werden. In diesem Fall findet die Inflation auf den entsprechenden Finanzmärkten oder dem Immobilienmarkt statt, ohne dass sie in den Statistiken als Inflation gemessen wird. Sie äußert sich dann in Form von steigenden Aktienkursen oder steigenden Grundstückpreisen, da die Nachfrage nach diesen Aktiven ansteigt, ohne dass sich das Angebot verändert. Solche Preissteigerungen haben häufig spekulativen Charakter. Bei länger anhaltenden, starken Preissteigerungen spricht man von spekulativen Blasen. Diese Blasen platzen oft abrupt (es kommt zum Crash) und verursachen dann Finanzkrisen.

In der Realität können wir alle der drei beschriebenen Auswirkungen beobachten, wobei je nach wirtschaftlicher Situation die eine oder andere dominiert. So war in Deutschland in den 1950er und 1960er Jahren in der Zeit des Wiederaufbaus und des Deutschen Wirtschaftswunders eindeutig die produktive Verwendung von Krediten vorherrschend. Geldschöpfung bewirkte vor allem reales wirtschaftliches Wachstum. Umgekehrt dominierte dort nach dem Ersten Weltkrieg bis zur Hyperinflation im Jahre 1923 die nicht produktive Verwendung von Geld, wie dies immer der Fall ist, wenn zu schnell zu viel Geld geschaffen wird. Der Börsenboom Ende der 1990er Jahre in den USA, aber auch in europäischen Ländern, ist wiederum ein Beispiel, wie neu geschaffenes Geld zu einem großen Teil zum Kauf von Aktien verwendet wurde und zu steigenden Börsenkursen führte.

In den meisten Zeiten haben wir eine Mischung von realem Wirtschaftswachstum und Inflation, da produktive und nicht-produktive Verwendung von neu geschaffenem Geld parallel stattfinden. Die spekulativen Auswirkungen der Geld- 
schöpfung auf den Finanzmärkten waren aber in der Vergangenheit meist auf bestimmte Episoden beschränkt (zum Beispiel der Börsenboom und nachfolgende Crash in der zweiten Hälfte der 1920er Jahre in den USA) und konnten in normalen Zeiten vernachlässigt werden. In neuester Zeit aber werden auch diese Auswirkungen zunehmend zu einem Dauerphänomen. Aus diesem Grund gewinnt die Verwendung von neu geschaffenem Geld auf Finanz- und Grundstückmärkten immer mehr an Bedeutung.

Keine der drei beschriebenen Auswirkungen folgt allein zwingend aus der Geldschöpfungstätigkeit. Geldschöpfung führt weder automatisch zu Wirtschaftswachstum noch zu Inflation noch zu spekulativen Blasen. Sie kann die eine oder die andere Auswirkung haben. Unabhängig davon, welche Auswirkung gerade dominiert, gilt aber die Tatsache, dass keine der drei Auswirkungen längerfristig ohne entsprechende Geldschöpfung möglich ist. Geldschöpfung ist eine notwendige, aber keine hinreichende Bedingung für Wirtschaftswachstum, für Inflation oder für spekulative Blasen.

Dabei gilt es zu beachten, dass die drei möglichen Auswirkungen der Geldschöpfung miteinander zusammenhängen und sich zum Teil gegenseitig bedingen. So wird ein Unternehmen möglicherweise gerade deshalb produktive Investitionen vornehmen, weil darauf spekuliert wird, dass Konsumenten verstärkt Konsumkredite aufnehmen und deshalb mehr kaufen. Oder es werden vermehrt Aktien auf Kredit an der Börse gekauft, weil man glaubt, dass in der Realwirtschaft neue produktive Investitionen finanziert werden, die einen neuen Boom auslösen.

Es gibt aber auch negative Zusammenhänge zwischen den verschiedenen Auswirkungen der Geldschöpfung. Werden etwa zu schnell zu viele Kredite vergeben, dann dominiert die nicht-produktive Verwendung des damit geschaffenen Geldes und die Folge davon ist eine rasche Zunahme der Inflation. Diese hat aber eine abschreckende Wirkung auf Investitionen in Maschinen und Anlagen, denn in solchen Fällen besteht Unsicherheit über den Realwert zukünftiger erwarteter Erträge von Investitionen. Stattdessen wird das Geld dann oft im Ausland angelegt, wo die Geldwertstabilität eher gewährleistet ist. Dies war vor allem der Fall während sogenannter Hyperinflationen wie zu Beginn der zoer Jahre des vergangenen Jahrhunderts in Deutschland.

Auch muss berücksichtigt werden, dass Geld immer wieder für andere Zwecke verwendet wird. Gehen wir beispielsweise davon aus, dass in einer Wirtschaft in einem bestimmten Jahr die Geldmenge über einen Kredit an ein Unternehmen erhöht wird. Das Unternehmen verwendet diesen Kredit produktiv, indem damit Investitionen in neues Realkapital finanziert werden. Am Ende des Jahres wird der Kredit zurückbezahlt und im nächsten Jahr vergibt die Bank einen Kredit in gleicher Höhe an ein anderes Unternehmen, welches diesen Kredit aber nicht produktiv, sondern zum Kauf von Aktien verwendet. In diesem Fall verändert sich die Geldmenge zwischen diesem und dem nächsten Jahr nicht und es lässt sich in der 
Statistik keine Zunahme der Geldmenge erkennen. Was sich ändert, ist aber die Auswirkung der Geldschöpfung. Ein Teil der Geldmenge, der in diesem Jahr produktiv verwendet wurde, wird im nächsten Jahr zum Kauf von Aktien verwendet. Es wird weniger in Maschinen und Anlagen investiert, aber dafür werden mehr Aktien gekauft.

Seit englische Goldschmiede im 17. Jahrhundert die bahnbrechende Entdeckung machten, dass Geld durch die Vergabe von Krediten aus dem Nichts geschaffen werden kann, kam es immer wieder auch zu Übertreibungen bei Kreditvergabe und Geldschöpfung. Eine exzessive Kreditvergabe rächt sich aber längerfristig. Das gilt zum einen für die Geschäftsbanken selbst, wenn ein stets größerer Teil der Kreditempfänger die erhaltenen Kredite nicht mehr zurückzahlen kann. Wird dies allgemein bekannt, leidet schnell auch das Vertrauen in das von den betroffenen Banken geschaffene Geld. Es kommt zu sogenannten BankRuns, bei welchen viele Kunden gleichzeitig das von Geschäftsbanken geschaffene Geld in rechtes Geld umtauschen wollen. Bis ins 19. Jahrhundert bedeutete dies, dass die Bankkunden versuchten, die von einer Geschäftsbank herausgegebenen Banknoten in Gold umzutauschen. Seit dem 20. Jahrhundert geht es hingegen darum, das auf den Konten liegende Geld abzuheben und damit das von einer Geschäftsbank geschaffene Giralgeld in von der Zentralbank stammende Banknoten umzuwandeln oder es auf Konten von anderen Geschäftsbanken zu transferieren, die weiterhin als sicher gelten. In beiden Fällen benötigt die Geschäftsbank große Mengen an zusätzlichen Reserven, entweder für den Bezug von weiterem Bargeld von der Zentralbank oder um die Geschäftsbanken zu bezahlen, auf deren Konten die Kunden jetzt ihre Gelder überweisen. Die Reserven einer Geschäftsbank sind aber begrenzt. Bei einem Bank-Run ist sie deshalb bald einmal nicht mehr in der Lage, ihre Zahlungsverpflichtungen zu erfüllen und wird zahlungsunfähig. Nur die Zentralbank kann sie dann noch vor dem Zusammenbrauch retten, indem sie ihr als sogenannter »Lender of Last Resort « unbegrenzt Reserven zur Verfügung stellt. Oder die Banken werden verstaatlicht, was in den letzten Jahren auch in mehreren Ländern praktiziert wurde.

Zum andern führt exzessive Kreditvergabe zu Inflation in der Realwirtschaft oder auf Finanzmärkten, was ebenfalls die wirtschaftliche Stabilität gefährdet. Denn je exzessiver die Kreditvergabe wird, umso weniger werden Kredite produktiv verwendet und umso häufiger kommt es dann zu Inflation und/oder zur Bildung spekulativer Blasen. Es kommt noch hinzu, dass spekulative Blasen an der Börse oder bei Immobilien häufig einen selbstverstärkenden Effekt auf die Kreditvergabe haben. Je mehr Aktien oder Immobilien an Wert gewinnen, umso leichter ist es, weitere Kredite von den Banken zu bekommen. Denn höhere Vermögenspreise bedeuten gleichzeitig auch höhere Sicherheiten, die man wiederum zum Erhalt von neuen Krediten verwenden kann. Steigende Vermögenswerte ermöglichen 
so mehr Geldschöpfung über Kredite, was zu weiter steigenden Vermögenswerten und weiterer Kreditvergabe führt.

Der Hang zum Exzess lässt sich in allen Phasen der Geschichte der Geldschöpfung seit dem 17. Jahrhundert beobachten. Das gilt auch für die uns bekannten spekulativen Blasen seit dem Ersten Weltkrieg. Sowohl der Börsenboom Ende der 1920er Jahre in den USA als auch die Blasen an verschiedenen Börsen in den 1990er Jahren waren mit exzessiver Kreditvergabe der Banken verbunden, die dann großenteils zu spekulativen Aktienkäufen verwendet wurden. Und die jüngste Finanzkrise von 2007 bis 2009 war das Resultat von exzessiver Vergabe von Hypothekarkrediten an nicht kreditwürdige Kunden (subprime loans), die zum Teil weder Vermögen noch Einkommen noch Jobs hatten. Möglich wurde dies dadurch, dass die Banken diese Hypothekarkredite verbrieften, das heißt Wertpapiere aus ihnen machten (mortgage backed securities) und sie dann an andere Investoren weiterverkauften.

Neben den Banken war es auch häufiger der Staat selbst, der für Exzesse bei der Geldschöpfung sorgte. Verursacht wurde dies in der Vergangenheit meist durch kriegerische Auseinandersetzungen, die einen kurzfristig hohen Finanzbedarf erzeugten. So kam es bereits Ende des 18. Jahrhunderts in England zu hohen Inflationsraten als Folge der Kredite, welche die Bank of England an den Staat zur Finanzierung der Napoleonischen Kriege vergab. Die Finanzierung von Kriegen führt eben nur zu einem kleinen Teil zu produktiver Verwendung des durch Kredite geschaffenen Geldes. Der größte Teil führt einfach zu einem Anstieg der Nachfrage nach bestehenden Gütern und Dienstleistungen und damit zu Inflation.

Extreme Formen nahm die Geldschöpfung durch Verschuldung des Staates in Deutschland nach dem Ende des Ersten Weltkrieges an, als Deutschland zu Reparationszahlungen an die Siegermächte verpflichtet wurde, aber kaum mehr konvertibles Geld (Goldmark) hatte. Aus diesem Grund fehlte es in Deutschland selbst an Geld, welches die Zentralbank der Wirtschaft zur Verfügung stellte, indem sie immer mehr Staatsschulden diskontierte. Diese Entwicklung mündete in die berühmte Deutsche Hyperinflation des Jahres 1923, als die Preise um das 100 Milliardenfache anstiegen. Dieser Exzess in der Geldschöpfung wurde bis heute nur noch durch die Inflation in Ungarn nach dem Zweiten Weltkrieg übertroffen. Nie zuvor hatte eine Zentralbank in so kurzer Zeit dermaßen viele Banknoten in Umlauf gebracht. Ähnliche durch den Staat verursachte Exzesse der Geldschöpfung wiederholten sich dann später in den 7oer und 8oer Jahren des vergangenen Jahrhunderts in mehreren lateinamerikanischen Ländern, wo ebenfalls Inflationsraten von mehreren hundert Prozent pro Jahr zu verzeichnen waren. Der Grund war dann nicht mehr die Finanzierung von Kriegen gegen andere Länder, sondern wie schon 1923 in Deutschland die Finanzierung der Begleichung von Schulden im Ausland.

Der kurze Abriss über die Geschichte der Exzesse in der Geldschöpfung zeigt, dass die produktive Verwendung von Krediten immer wieder in den Hintergrund 
tritt. Stattdessen dominiert dann die nicht-produktive Verwendung von Krediten zur Finanzierung von Käufen in der Realwirtschaft, ohne dass die Produktion ausgeweitet wird, oder zur Finanzierung von Wertpapieren oder Immobilien. Ersteres ist oft auf eine exzessive Monetisierung von Staatsschulden zurückzuführen. In diesem Fall kaufen Banken Staatsschulden auf, was in der Wirtschaft zu einer Zunahme der Geldmenge führt, ohne dass mehr produziert wird. Staatlich initiierte Geldschöpfungsexzesse sind in letzter Zeit jedoch relativ selten. In funktionierenden Volkswirtschaften können Hyperinflationen inzwischen vermieden werden, solange die Zentralbanken als von der Regierung unabhängige Instanzen die Inflation im Zaum halten. Ganz anders verhält es sich mit der Verwendung von Krediten zur Finanzierung von Käufen von Wertpapieren und Immobilien. In den letzten Jahrzehnten ließen sich spekulative Blasen immer häufiger beobachten. Sie sind mittlerweile zum ökonomischen Alltag geworden und der Einfluss der Geldschöpfung auf Aktienkurse oder Immobilienpreise ist während dieser Episoden oftmals stärker ausgeprägt als der Einfluss auf das reale Wirtschaftswachstum oder auf das Preisniveau.

\section{Wo stehen wir heute? Die Finanzkrise von 2007/2008 und ihre Folgen}

Betrachten wir die jüngste Zeit seit der Finanzkrise von 2007/2008, dann lässt sich ein weitgehender Kontrollverlust der Zentralbanken über den Prozess der Geldschöpfung feststellen. In den USA, England und der Schweiz haben die Geschäftsbanken dank massiven Kaufprogrammen der Zentralbanken seither enorme Mengen von Reserven zur Verfügung, von denen sie nur einen Bruchteil effektiv für die Geldschöpfung brauchen. Kurzfristige Refinanzierungsgeschäfte, über welche die Zentralbanken bisher die Geschäftsbanken mit Reserven versorgten, finden deshalb seit 2009 kaum mehr statt und eine Geldpolitik im traditionellen Sinn gibt es nicht mehr. Zentralbanken haben nur direkten Einfluss auf die Tätigkeit der Geschäftsbanken, solange sie etwas besitzen, was diese auch brauchen. Wenn die Banken aber, wie dies heute der Fall ist, viel mehr Reserven haben, als sie zur Kreditvergabe brauchen, sind sie für die Geldschöpfung nicht mehr auf die Zentralbank angewiesen.

Ein Verlust über die Kontrolle der Geldschöpfung durch die Zentralbanken ist grundsätzlich nichts Neues. Solche Episoden konnten in der Geschichte immer wieder beobachtet werden. Die schon erwähnte Deutsche Hyperinflation zu Beginn der 2oer Jahre des vergangenen Jahrhunderts ist ein eindrückliches Beispiel für eine Episode völlig unkontrollierter Geldschöpfung. Den Zentralbanken gelang es allerdings jeweils, die Kontrolle über die Geldschöpfung mit mehr oder weniger 
schmerzvollen Eingriffen zurückzugewinnen, notfalls auch mit einer Währungsreform wie in Deutschland im Jahre 1923 oder nach dem Zweiten Weltkrieg.

Auch eine langfristige Analyse der Summe der Aktiven der acht weltweit wichtigsten Zentralbanken lässt die Zeitspanne von 2007 bis 2014 mit ihren Vervielfachungen der Aktiven noch nicht als historisch einmalig erscheinen. ${ }^{5}$ Setzen wir die Summe der Aktiven zum BIP in Bezug, dann zeigt sich, dass dieses Verhältnis im Jahr 2014 durchschnittlich ungefähr 30:1 betrug. In normalen Zeiten liegt dieses Verhältnis zwischen 10:1 und 20:1, doch am Ende des Zweiten Weltkrieges lag es sogar bei fast 40:1 und damit noch um einiges höher als heute. Dieses Rekordniveau wurde damals verursacht durch die massive Monetisierung von Staatsschulden bei den Zentralbanken. Um den Krieg zu finanzieren, emittierten die betroffenen Staaten Anleihen in großem Stil, die dann von der Zentralbank aufgekauft und auf diese Weise durch neu geschaffenes Zentralbankengeld finanziert wurden.

In der jüngsten Episode seit 2007 wurde die Expansion der Zentralbankenbilanzen hingegen nicht durch die Finanzierung von Schulden, sondern, wie weiter oben aufgezeigt, durch Rettungsmaßnahmen zur Erhaltung eines funktionierenden Finanzsystems ausgelöst. In beiden Fällen kam es unmittelbar zu einer massiven Zunahme der Reserven bei den Geschäftsbanken. Während des Zweiten Weltkrieges wurden diese Reserven von den Geschäftsbanken jedoch vor allem dazu genutzt, die Geldschöpfungstätigkeit weiter auszudehnen, während die Reserven in der jüngsten Episode von den Geschäftsbanken bisher kaum weiterverwendet wurden.

Obwohl also ein zeitweiser Kontrollverlust der Zentralbanken über die Geldschöpfung historisch gesehen nichts Besonderes ist und auch die Aktiven der Zentralbanken im Vergleich zum BIP gegenwärtig nicht auf einem Rekordniveau liegen, führt die jüngste Entwicklung in historisches Neuland. Dies liegt zum einen daran, dass die Kriterien zur Deckung des von der Zentralbank geschaffenen Geldes (Reserven und Bargeld) offiziell noch nie dermaßen locker waren und zum andern an einem historisch einmalig tiefen Zinsniveau. Die heutige Situation steht am Ende einer langen Entwicklung, die mit einem stets größer werdenden Vertrauen in das von den Banken geschaffene Geld verbunden ist. $\mathrm{Zu}$ Beginn, als Londoner Goldschmiede im 17. Jahrhundert zum ersten Mal Geldschöpfung betrieben, wurde den von den Goldschmieden herausgegebenen Banknoten nur getraut, weil man wusste, dass man sie notfalls in Gold umtauschen konnte. Diese Golddeckung wurde dann aber nach und nach immer mehr zur Fiktion und es genügte das Wissen, dass die Banknoten durch Anleihen von sicheren Schuldnern (vor allem Staatsschulden) gedeckt waren. 
Inzwischen ist aber ein großer Teil des Zentralbankengeldes nicht einmal mehr durch sichere Schulden gedeckt. In den Aktiven der Zentralbanken befinden sich heute auch Aktien oder Hypothekarforderungen. Die Aufweichung der Kriterien für Sicherheiten seit dem Jahr 2007 wurde aber kaum zur Kenntnis genommen. De facto spielt es heute keine Rolle mehr, was die Zentralbank den Geschäftsbanken abkauft, damit diese an zusätzliche Reserven gelangen. Entscheidend ist der Glaube an ein längerfristig weiterhin stattfindendes Wirtschaftswachstum und damit an die produktive Verwendung eines Teils der von den Banken vergebenen Kredite. Auf diese Weise wird sichergestellt, dass die Mehrheit der Schulden in einer Wirtschaft trotz zeitweiliger Wirtschaftskrisen langfristig doch zurückbezahlt werden können. Und dieser Glaube hat sich bis heute bestätigt.

Es scheint aber zweifelhaft, ob Zentralbanken je wieder zu einer normalen Geldpolitik zurückkehren können. Da spekulative Blasen auf Finanzmärkten in den letzten Jahren quasi zu einem Dauerphänomen geworden sind, wurden auch die saußergewöhnlichen Reaktionen` der Zentralbanken auf diese Phänomene letztlich zu einem Dauerphänomen. Ein stets größerer Teil des durch die Geschäftsbanken geschaffenen Geldes fließt heute nicht mehr in die Realwirtschaft, sondern auf den Immobilienmarkt oder auch an die Börse. Zwangsläufig kommt es dann immer wieder zu spekulativen Blasen auf diesen Märkten, ohne dass die Realwirtschaft in gleichem Ausmaß mitwächst. Diese Blasen platzen aber irgendwann und münden häufiger in Krisen, weil die auf Krediten aufbauenden Expansionen an ein Ende gelangen, wenn ein Teil der Kredite nicht mehr bedient werden kann.

Dies ist ganz besonders dann der Fall, wenn die Zentralbanken versuchen, die spekulativen Exzesse mit Zinssatzerhöhungen zu bremsen. Schon mehrere spekulative Blasen wie etwa die jüngste Immobilienblase in den USA und England im Jahre 2007 wurden so zum Platzen gebracht. Die dadurch ausgelösten Wirtschaftskrisen zwingen die Zentralbanken aber sofort dazu, die Zinsen wieder zu senken und die Geschäftsbanken großzügig mit Reserven zu versorgen, wenn sie einen Zusammenbruch des Finanzsystems verhindern wollen. Wenn Zentralbanken aber fast permanent Rettungsinterventionen vornehmen müssen, können sie gleichzeitig ihre Rolle als Kontrolleure des Geldschöpfungsprozesses kaum noch wahrnehmen.

\section{Literatur}

Angas, Lawrence Lee Bazley: Slump Ahead in Bonds, New York: Somerset Publishers 1937.

Binswanger, Mathias: Geld aus dem Nichts. Wie Banken Wachstum ermöglichen und Krisen verursachen, Weinheim: Wiley-VCH 2015. 
Deutsche Bundesbank: Geld und Geldpolitik, Frankfurt a.M.: Deutsche Bundesbank 2019, https://www.bundesbank.de/de/publikationen/schule-und-bildung /geld-und-geldpolitik-606038 (letzter Abruf 20.06.2020).

Ferguson, Niall/Schaab, Andreas/Schularick Moritz: Central Bank Balance Sheets. Expansion and Reduction since 1900, CESifo Working Paper No. 5379 (2015). McLeay, Michael/Radia, Amar/Thomas, Ryland: "Money Creation in the Modern Economy«, in: Bank of England Quarterly Bulletin Q1 (2014), S. 14-27. 
